\title{
Fronts in reactive convection: bounds, stability and instability
}

\author{
Peter Constantin* $\quad$ Alexander Kiselev ${ }^{\dagger} \quad$ Lenya Ryzhik ${ }^{\ddagger}$
}

October 31, 2018

\begin{abstract}
We consider front propagation in a reactive Boussinesq system in an infinite vertical strip. We establish nonlinear stability of planar fronts for narrow domains when the Rayleigh number is not too large. Planar fronts are shown to be linearly unstable with respect to long wavelength perturbations if the Rayleigh number is sufficiently large. We also prove uniform bounds on the bulk burning rate and the Nusselt number in the KPP reaction case.
\end{abstract}

\section{Introduction}

The presence of fluid flow may have a strong effect on reaction processes in many situations of interest [19, 28]. Many recent studies focused on the influence of passive advection on combustion. During passive advection the reacting material is carried by a flow, but the flow is not influenced by the reaction. Traveling waves and pulsating fronts in periodic flows have been studied in [3, 4, 2, 25, 26]; the thin front limit was considered in [11, 12, 17] using homogenization techniques. Estimates on the speed of front propagation for different classes of flows have been derived in [1, 5, 16, 6, 13. Numerical studies of the effect of a passive flow on combustion were carried out in [14, 15, 8, 9, 23]. This list is far from complete; we refer to the recent reviews [1, 27] for further references.

However, there have been few rigorous works on models with feedback of the chemical reaction on the flow field. In this paper we study a simplified active combustion model [18, 22, 20, 21] in which the reaction does influence the flow. The feedback of the flame on the fluid is taken in a Boussinesq approximation. The model couples thus an advectiondiffusion-reaction equation for the temperature with an incompressible Navier-Stokes system driven by temperature differences. We study this problem in a two dimensional strip of infinite vertical height and finite horizontal width. The vertical direction is the direction of gravity. The system admits planar fronts as particular solutions. These fronts correspond to traveling solutions of the one dimensional reaction-diffusion system without horizontal

\footnotetext{
*Department of Mathematics, University of Chicago, Chicago, IL 60637, const@math.uchicago.edu

${ }^{\dagger}$ Department of Mathematics, University of Wisconsin, Madison, WI 53706, kiselev@math.wisc.edu

${ }_{\ddagger}^{\ddagger}$ Department of Mathematics, University of Chicago, Chicago, IL 60637, ryzhik@math.uchicago.edu
} 
variation. We study them in the context of the larger reactive Boussinesq system. Coupling with the Boussinesq system introduces at least two new interesting effects. One is symmetry breaking: gravity breaks the vertical symmetry of the reaction diffusion systems. This has a dynamical effect: fronts connecting low regions of hot fluid to high regions of cold fluid are susceptible to the Rayleigh-Taylor instability [7]. The second effect is due to the introduction of new horizontal degrees of freedom: new length scales are introduced. When one ignores the fluid advection, the planar fronts have a characteristic thickness $\delta$ (2.11), which is determined by the thermal diffusivity and the characteristic reaction time $t_{c}(2.13)$. Using these as length and time units, three significant nondimensional parameters emerge. One is the aspect ratio $\lambda(2.23)$, the ratio of the horizontal width of the strip to the thickness of the planar front. The second parameter is the Prandtl number $\sigma$ (2.19), the ratio of kinematic viscosity to thermal diffusivity. The third important parameter is the Rayleigh number $\rho(2.20)$ which measures the relative strength of the buoyancy force on the scale of the front thickness.

We prove three kinds of results: stability, instability and uniform bounds. Stability is analyzed in Section 4 . If the aspect ratio $\lambda$ is small, then the only traveling solutions are planar fronts, and all solutions become eventually planar. This is a consequence of the fact that diffusion acts rapidly across a narrow strip. This stability mechanism is quite robust and operates for all kinds of nonlinearities. If the Rayleigh number is large enough, then the planar front loses stability to long wave perturbations, which are present if the aspect ratio is large enough. The instability is of Rayleigh-Taylor type and is also expected to be quite robust. We note that nonplanar traveling waves have been recently shown to exist ( 20, 21]) in reactive Boussinesq systems with bistable nonlinearity at large enough Rayleigh numbers. Our results on planar front instability agree qualitatively with recent numerical results [22]. The proof appears in Section 5 and is based on the intuitive idea that, in the presence of gravity, the neutral mode - corresponding to the broken vertical translation symmetry - misaligns over long horizontal distances, and gives rise to a long wave unstable mode. Technically, one needs to deal with a variable coefficient linear operator in which the instability of heavy fluid on top of light fluid is exploited using the monotonicity of the front. In Section 3 we prove bounds for arbitrary solutions of front type. The results state that the bulk burning rate, speed of front, gradients of temperature, and fluid quantities, are all bounded. The bounds do depend on the aspect ratio. The proof applies only to concave KPP nonlinearities. The results imply that there are no accelerating fronts in this system. The proof of the upper bound passes via a lower bound: the temperature gradients squared bound from below the bulk burning rate [5]. On the other hand, the bulk burning rate is bounded above by the sum of laminar burning rate and temperature gradients to power one. This implies that the temperature gradients are bounded, and the rest follows.

\section{Reactive Boussinesq fronts}

The reactive Boussinesq equations are

$$
\begin{gathered}
\frac{\partial v}{\partial t}+v \cdot \nabla v+\nabla p-\nu \Delta v=g A T e_{z} \\
\nabla \cdot v=0
\end{gathered}
$$




$$
\frac{\partial T}{\partial t}+v \cdot \nabla T-\kappa \nabla T=\frac{v_{0}^{2}}{4 \kappa} f(T) .
$$

Here $v(\mathbf{x}, t)=(u(\mathbf{x}, t), w(\mathbf{x}, t))$ and $T(\mathbf{x}, t)$ are the velocity and the (normalized) temperature. The vector $e_{z}$ is the unit vector pointing in the direction opposite to the pull of gravity. The constants $\nu, \kappa, A, v_{0}, g$ are all positive, and represent, respectively, the kinematic viscosity, thermal diffusivity, thermal expansion coefficient multiplied by the temperature variation scale, speed of purely reactive-diffusive front and acceleration of gravity. The variables $\mathbf{x}=$ $(x, z)$ belong to a strip, with $z \in \mathbb{R}$ and $x \in[0, L]$. The boundary conditions for the normalized temperature are front conditions,

$$
T(x, z, t) \rightarrow 1 \quad \text { as } z \rightarrow-\infty, \quad T(x, z, t) \rightarrow 0 \text { as } z \rightarrow \infty .
$$

We assume throughout the paper that the initial condition $T_{0}(x, z)$ satisfies $0 \leq T_{0}(x, z) \leq 1$. The velocity vanishes at the two ends of the strip:

$$
v(x, z, t) \rightarrow 0 \text { as }|z| \rightarrow \infty
$$

The lateral boundary conditions are periodic:

$$
T(x+L, z, t)=T(x, z, t), \quad v(x+L, z, t)=v(x, z, t) .
$$

We consider the vorticity

$$
\omega(x, z, t)=\frac{\partial w}{\partial x}-\frac{\partial u}{\partial z}
$$

where $u$ is the component of $v$ in the $x$ direction and $w$ is the component of $v$ in the $z$ direction. The momentum equation (2.1) implies

$$
\frac{\partial \omega}{\partial t}+v \cdot \nabla \omega-\nu \Delta \omega=g A \frac{\partial T}{\partial x}
$$

The nonlinearity $f(T)$ satisfies $f(0)=f(1)=0$, is a smooth function and is assumed to be of one of the following three types: bistable, ignition type or concave KPP. Bistable nonlinearities are such that there exists $\theta \in(0,1)$ so that $f(T)<0$ for $T \in(0, \theta)$ and $f(T)>0$ for $T \in(\theta, 1)$. Ignition type nonlinearities are such that there exists $\theta \in(0,1)$ so that $f(T)=0$ for $T \in[0, \theta]$ and $f(T)>0$ for $T \in(\theta, 1)$. Concave KPP type nonlinearities have $f(T)>0$ for $T \in(0,1)$ and $f^{\prime \prime}(T)<-M$ for $T \in[0,1]$ with $M>0$. In their case we assume that the slope at zero is positive and normalized, $f^{\prime}(0)=1$, while the slope at 1 is negative, $f^{\prime}(1)=-m<0$. We will use the KPP properties only in the proof of a uniform upper bound for the burning rate presented in Section 3. We summarize the essential properties of the KPP reaction used in that proof:

$$
0 \leq f(T) \leq T,-f^{\prime \prime}(T) \geq M>0,-f^{\prime}(T) \leq m, \text { for } 0 \leq T \leq 1
$$

The Boussinesq system has flat traveling wave solutions

$$
T_{f r}=\tau(z-c t), v_{f r}=0 .
$$

The momentum equation (2.1) holds in this case because the pressure can balance a temperature that depends on $z$ and $t$ alone. The speed $c$ takes all values $c \geq v_{0}$ in the KPP case, 
and is unique in the bistable and ignition case. The profile $\tau(z)$ is monotonically decreasing in all three cases, and obeys

$$
\kappa \tau^{\prime \prime}+c \tau^{\prime}+\frac{v_{0}^{2}}{4 \kappa} f(\tau)=0
$$

where $\tau^{\prime}=\frac{d \tau}{d z}$. We choose now dimensional units. First we note that the function $\tau$ varies on length scale of the order

$$
\delta=\frac{2 \kappa}{v_{0}}
$$

that is, the function $\tau$ has the form $\tau(z)=P(z / \delta)$, where $P$ obeys the equation

$$
P^{\prime \prime}+2 \bar{c} P^{\prime}+f(P)=0 .
$$

Here $\bar{c}=c / v_{0}$ is a nondimensional constant that is determined solely by the form of the nonlinearity $f(T)$, and that in turn determines the traveling front speed $c=\bar{c} v_{0}$ in the original dimensional variables. We choose reaction units: we take (2.11) for length unit and we take

$$
t_{c}=\frac{4 \kappa}{v_{0}^{2}}
$$

for time unit. The velocity fluctuations are written as

$$
v(\mathbf{x}, t)=\frac{\delta}{t_{c}} \tilde{v}\left(\frac{\mathbf{x}}{\delta}, \frac{t}{t_{c}}\right)
$$

and the temperature fluctuations are written as

$$
\theta(\mathbf{x}, t)=\tilde{\theta}\left(\frac{\mathbf{x}}{\delta}, \frac{t}{t_{c}}\right)
$$

Using these units, rescaling, using $\mathbf{x}=(x, z)=\left(x_{\text {new }}, z_{\text {new }}\right)=\left(x_{\text {old }} / \delta, z_{\text {old }} / \delta\right)$ and $t=t_{\text {new }}=$ $t_{\text {old }} / t_{c}$, and dropping tildes, we derive the nonlinear equations

$$
\frac{\partial \omega}{\partial t}+v \cdot \nabla \omega-\sigma \Delta \omega=\sigma \rho \frac{\partial T}{\partial x}
$$

and

$$
\frac{\partial T}{\partial t}+v \cdot \nabla T-\Delta T=f(T)
$$

where $v=(u, w)$, with

$$
\Delta u=-\frac{\partial \omega}{\partial z}, \quad \Delta w=\frac{\partial \omega}{\partial x}
$$

which follows from (2.7) and incompressibility. The nondimensional parameters are the Prandtl number

$$
\sigma=\frac{\nu}{\kappa}
$$

and the Rayleigh number across a laminar front width

$$
\rho=\frac{g A \delta^{3}}{\kappa \nu} .
$$


The boundary conditions in $z$ are of front type

$$
\left\{\begin{array}{l}
T(x, z, t) \rightarrow 1 \text { as } z \rightarrow-\infty \\
T(x, z, t) \rightarrow 0 \text { as } z \rightarrow+\infty \\
v(x, z, t) \rightarrow 0, \text { as }|z| \rightarrow \infty \\
\omega(x, z, t) \rightarrow 0 \text { as }|z| \rightarrow \infty
\end{array}\right.
$$

The boundary conditions in $x$ are periodic

$$
T(x+\lambda, z, t)=T(x, z, t), \quad v(x+\lambda, z, t)=v(x, z, t), \omega(x+\lambda, z, t)=\omega(x, z, t)
$$

with period

$$
\lambda=\frac{L}{\delta}
$$

\section{General bounds in the KPP case}

We study general solutions of the system (2.16, 2.17, 2.18) with a KPP nonlinearity that satisfies assumptions (2.9), and with front-like initial data: functions that approach the values 0 as $z \rightarrow \infty$ and, respectively 1 as $z \rightarrow-\infty$ at least at an exponential rate. We denote $D=[0, \lambda] \times \mathbb{R}$. We will use the notation

$$
\|g\|_{L^{2}}^{2}=\frac{1}{\lambda} \int_{D}|g(x, z)|^{2} d x d z
$$

for the normalized $L^{2}$ norm. We consider the bulk burning rate

$$
V(t)=\frac{1}{\lambda} \int_{D} \frac{\partial T(x, z, t)}{\partial t} d x d z
$$

as a measure of the reaction rate. Using equation (2.17), incompressibility of $u$ and the boundary conditions for $u$ and $T$ as $z \rightarrow \pm \infty$ we have also

$$
V(t)=\frac{1}{\lambda} \int_{D} f(T(x, z, t)) d x d z
$$

The time average of $V$ is denoted $\bar{V}$

$$
\bar{V}(t)=\frac{1}{t} \int_{0}^{t} V(s) d s .
$$

Lemma 1 Assume that there exists a constant $\alpha \in \mathbb{R}$ so that the front-like initial data $T_{0}(x, z)$ obeys

$$
T_{0}(x, z) \leq \exp (\alpha-z)
$$

and

$$
\left(1-T_{0}(x, z)\right) \leq \exp (\alpha+z)
$$


Then the solution of (2.17) obeys the bounds

$$
T(x, z, t) \leq \exp \left[\alpha-z+2 t+\int_{0}^{t}\|w(\cdot, s)\|_{L^{\infty}} d s\right]
$$

and

$$
(1-T(x, z, t)) \leq \exp \left[\alpha+z+t-\int_{0}^{t}\|w(\cdot, s)\|_{L^{\infty}} d s\right]
$$

for all $t \geq 0$.

Proof. For the bound of $T$ we seek a supersolution of the form:

$$
\theta_{+}(z, t)=\exp \left[-a z+a \int_{0}^{t}\|w(\cdot, s)\|_{L^{\infty}} d s+b t+\alpha\right], \quad a>0 .
$$

Using (2.9) we get

$$
\frac{\partial \theta_{+}}{\partial t}+v \cdot \nabla \theta_{+}-\Delta \theta_{+}-f\left(\theta_{+}\right) \geq 0
$$

if $b \geq a^{2}+1$. We chose for simplicity of exposition $a=1$, and took the most economic $b=2$. For the bound of $1-T$ we seek a subsolution for $T$ of the form

$$
\theta_{-}(z, t)=1-\exp \left[a z-a \int_{0}^{t}\|w(\cdot, s)\|_{L^{\infty}} d s+b t+\alpha\right]
$$

and, using the fact that $f \geq 0$ on $[0,1]$, the condition

$$
\frac{\partial \theta_{-}}{\partial t}+v \cdot \nabla \theta_{-}-\Delta \theta_{-}-f\left(\theta_{-}\right) \leq 0
$$

follows if

$$
b \geq a^{2} .
$$

Again, we chose $a=1$ for simplicity, and took the best $b=1$. The proof shows that similar inequalities hold if the exponential rate of decay at infinity of the initial data is different than $a=1$, or if the initial data approaches its limit at negative infinity at a different exponential rate than $a=1$.

Let us consider the average quantities

$$
W(t)=\frac{1}{t} \int_{0}^{t}\|w(\cdot, s)\|_{L^{\infty}} d s
$$

and

$$
N(t)=\frac{1}{t} \int_{0}^{t}\|\nabla T(\cdot, s)\|_{L^{2}}^{2} d s
$$

representing the average maximum vertical velocity and temperature gradient squared.

Lemma 2 Consider front-like initial data that satisfy (3.5). Then the solutions of (2.17) obey

$$
\bar{V}(t) \leq W(t)+2+\frac{\gamma}{t}
$$

for all $t \geq 0$ with $\gamma$ depending on the initial data. 
Proof. First we write

$$
\bar{V}(t)=\frac{1}{\lambda t} \int_{D}\left(T(x, z, t)-T_{0}(x, z)\right) d x d z
$$

which we bound as

$$
\bar{V}(t) \leq \frac{1}{\lambda t} \int_{0}^{\lambda} d x\left[\int_{-\infty}^{0}\left(1-T_{0}(x, z)\right) d z+\int_{0}^{\infty} T(x, z, t) d z\right]
$$

using the fact that $T(t, x, z) \leq 1$. Now, denoting

$$
B_{1}(t)=\alpha+2 t+\int_{0}^{t}\|w(\cdot, s)\|_{L^{\infty}} d s
$$

we have from $(3.7)$ that

$$
\int_{B_{1}(t)}^{\infty} T(x, z, t) d z \leq 1
$$

while, because $T \leq 1$, we have

$$
\int_{0}^{B_{1}(t)} T(x, z, t) d z \leq B_{1}(t)
$$

We use these bounds in (3.12) and obtain (3.11). Now we bound $W(t)$ below by $N(t)$ :

Lemma 3 Consider front-like initial data that satisfy (3.5). Then the solutions of (2.17) obey

$$
N(t) \leq \frac{m+2}{M} W(t)+\frac{2 m+3}{M}+\frac{\Gamma}{t}
$$

with $m$ and $M$ given in (2.9) and with $\Gamma$ depending on the initial data.

Proof. We start by computing

$$
\frac{d}{d t} V(t)=\frac{1}{\lambda} \int_{D} f^{\prime}(T(x, z, t)) \frac{\partial T(x, z, t)}{\partial t} d x d z
$$

Using (2.17) and integrating by parts, using incompressibility of $u$ and the boundary conditions, we obtain

$$
\frac{d V}{d t}-\frac{1}{\lambda} \int_{D} f^{\prime}(T(x, z, t)) f(T(x, z, t)) d x d z=-\frac{1}{\lambda} \int_{D} f^{\prime \prime}(T(x, z, t))|\nabla T(x, z, t)|^{2} d x d z .
$$

Using (2.9) we deduce

$$
\frac{d V}{d t}+m V(t) \geq M\|\nabla T(\cdot, t)\|_{L^{2}}^{2}
$$

Taking a time average we get

$$
\frac{1}{t}(V(t)-V(0))+m \bar{V}(t) \geq M N(t) .
$$


We observe that

$$
\begin{aligned}
V(t) & =\frac{1}{\lambda} \int_{D} f(T(x, z, t)) d x d z=\int_{0}^{\lambda} \frac{d x}{\lambda} \int_{-\infty}^{-B_{2}(t)} f(T(x, z, t)) d z \\
& +\int_{0}^{\lambda} \frac{d x}{\lambda} \int_{-B_{2}(t)}^{B_{1}(t)} f(T(x, z, t)) d z+\int_{0}^{\lambda} \frac{d x}{\lambda} \int_{B_{1}(t)}^{\infty} f(T(x, z, t)) d z
\end{aligned}
$$

where $B_{1}(t)$ is given in $(3.13)$ and

$$
B_{2}(t)=\alpha+t+\int_{0}^{t}\|w(\cdot, s)\|_{L^{\infty}} d s .
$$

We use the inequality $f(T) \leq m(1-T)$ which follows from (2.9). Then we have

$$
\int_{0}^{\lambda} \frac{d x}{\lambda} \int_{-\infty}^{-B_{2}(t)} f(T(x, z, t)) d z \leq m,
$$

as follows from (3.8). Similarly,

$$
\int_{0}^{\lambda} \frac{d x}{\lambda} \int_{B_{1}(t)}^{\infty} f(T(x, z, t)) d z \leq 1
$$

follows from (3.7) and (2.9). The second term on the right in (3.16) is bounded by $B_{1}(t)+$ $B_{2}(t)$. Thus, returning to (3.15), we have

$$
M N(t) \leq m \bar{V}(t)+3+2 W(t)+\frac{c}{t} .
$$

In view of (3.11) of the previous lemma, (3.14) is proven.

The next step consists in bounding the quantity $W(t)$ in terms of $N(t)$, using the vorticity equation (2.16).

Lemma 4 There exists an absolute constant $C$ so that, for all $t>0$ one has

$$
W(t) \leq C \lambda^{3 / 2}\left\{\rho \lambda \sqrt{N(t)}+\frac{1}{\sqrt{\sigma t}}\left\|\omega_{0}\right\|_{L^{2}}\right\},
$$

where $\omega_{0}(x, z)$ is the initial data for $\omega(x, z, t)$.

Proof. We multiply (2.16) by $\omega$ and integrate. After one integration by parts we obtain

$$
\frac{1}{2} \frac{d}{d t} \int_{D}|\omega(x, z, t)|^{2} \frac{d x d z}{\lambda}+\sigma \int_{D}|\nabla \omega(x, z, t)|^{2} \frac{d x d z}{\lambda}=\sigma \rho \int_{D} \omega(x, z, t) \frac{\partial T(x, z, t)}{\partial x} \frac{d x d z}{\lambda} .
$$

We introduce

$$
\bar{T}(z, t):=\int_{0}^{\lambda} T(x, z, t) \frac{d x}{\lambda}
$$

and note the obvious fact that

$$
\frac{\partial T(x, z, t)}{\partial x}=\frac{\partial(T(x, z, t)-\bar{T}(z, t))}{\partial x} .
$$


Inserting this expression into the right hand side of (3.18) and integrating by parts on the right side we obtain

$$
\begin{aligned}
& \frac{1}{2} \frac{d}{d t} \int_{D}|\omega(x, z, t)|^{2} \frac{d x d z}{\lambda}+\sigma \int_{D}|\nabla \omega(x, z, t)|^{2} \frac{d x d z}{\lambda} \\
& =-\sigma \rho \int_{D} \frac{\partial \omega(x, z, t)}{\partial x}(T(x, z, t)-\bar{T}(z, t)) \frac{d x d z}{\lambda}
\end{aligned}
$$

Using Young's inequality together with the inequality

$$
\int_{D}|T(x, z, t)-\bar{T}(z, t)|^{2} \frac{d x d z}{\lambda} \leq \lambda^{2} \int_{D}|\nabla T(x, z, t)|^{2} \frac{d x d z}{\lambda}
$$

we deduce

$$
\begin{aligned}
& \frac{1}{2} \frac{d}{d t} \int_{D}|\omega(x, z, t)|^{2} \frac{d x d z}{\lambda}+\sigma \int_{D}|\nabla \omega(x, z, t)|^{2} \frac{d x d z}{\lambda} \\
& \leq \frac{\sigma}{2} \int_{D}\left|\frac{\partial \omega(x, z, t)}{\partial x}\right|^{2} \frac{d x d z}{\lambda}+\frac{\sigma \rho^{2} \lambda^{2}}{2} \int_{D}|\nabla T(x, z, t)|^{2} \frac{d x d z}{\lambda}
\end{aligned}
$$

Integrating (3.19) in time we deduce

$$
\frac{1}{t} \int_{0}^{t} d s \int_{D}|\nabla \omega(x, z, s)|^{2} \frac{d x d z}{\lambda} \leq \rho^{2} \lambda^{2} N(t)+\frac{1}{\sigma t}\left\|\omega_{0}\right\|_{L^{2}}^{2}
$$

Let us represent the function $w$ in terms of its Fourier series

$$
w(x, z, t)=\sum_{k \in \frac{2 \pi}{\lambda} \mathbf{Z}} w_{k}(z, t) e^{i k x}
$$

and note that, in view of incompressibility, $w_{0}(z, t)$ is independent of $z$, and hence the boundary conditions at $z \pm \infty$ imply that

$$
w_{0}(z, t)=0 .
$$

In view of (2.18), the embedding inequality

$$
\|w(\cdot, t)\|_{L^{\infty}} \leq C \lambda^{3 / 2}\|\nabla \omega(\cdot, t)\|_{L^{2}}
$$

follows. The constant $C$ is absolute (recall that the $L^{2}$ norm is normalized by $\lambda(3.1)$ ); it can be computed either by using Parseval's identity or using (5.16) below. From (3.21) and (3.20) we deduce (3.17), using the Cauchy-Schwartz inequality for integration in time.

Theorem 1 Solutions of (2.10), (2.17), 2.18) with front-like initial data obey

$$
\begin{gathered}
N(t) \leq \frac{C^{2}(m+2)^{2}}{M^{2}} \rho^{2} \lambda^{5}+\frac{4 m+6}{M}+\left(\frac{K_{1}}{\sqrt{t}}+\frac{K_{2}}{t}\right), \\
W(t) \leq \frac{C^{2}(m+2)}{M} \rho^{2} \lambda^{5}+C \sqrt{\frac{4 m+6}{M}} \rho \lambda^{5 / 2}+\frac{K_{3}}{t^{\frac{1}{4}}}+\frac{K_{4}}{t^{\frac{1}{2}}}
\end{gathered}
$$


and

$$
\limsup _{t \rightarrow \infty} \bar{V}(t) \leq 2+\frac{C^{2}(m+2)}{M} \rho^{2} \lambda^{5}+C \sqrt{\frac{4 m+6}{M}} \rho \lambda^{5 / 2}
$$

with $M, m$ given in (2.9), $C$ the absolute constant of (3.21) and with $K_{1}, K_{2}, K_{3}, K_{4}$ depending on the initial data.

Proof. We insert (3.17) into the right hand side of (3.14). We obtain

$$
N(t) \leq \frac{m+2}{M} C \rho \lambda^{5 / 2} \sqrt{N(t)}+\frac{C \lambda^{3 / 2}(m+2)}{M \sqrt{\sigma t}}\left\|\omega_{0}\right\|_{L^{2}}+\frac{2 m+3}{M}+\frac{\Gamma}{t} .
$$

Now we use Young's inequality

$$
\frac{m+2}{M} C \rho \lambda^{5 / 2} \sqrt{N(t)} \leq \frac{N(t)}{2}+\frac{C^{2}(m+2)^{2}}{2 M^{2}} \rho^{2} \lambda^{5}
$$

and deduce (3.22). Then (3.23) follows from (3.22) and (3.17), while (3.24) follows from (3.23) and (3.11).

Remarks. 1. In terms of the original dimensional variables $x_{\text {old }}$ of $(2.1),(2.2),(2.3)$ the bound on the wave number (3.22) means that the Nusselt number on scale $L$

$$
N u=\lim \sup _{t \rightarrow \infty} \frac{1}{t} \int_{0}^{t} \int_{0}^{L} \int_{-\infty}^{\infty}|\nabla T(x, z, s)|^{2} d x d z d s=\lambda \lim \sup _{t \rightarrow \infty} N(t)
$$

is bounded as

$$
N u \leq \frac{C^{2}(m+2)^{2}}{M^{2}} R a^{2}+\frac{4 m+6}{M} \lambda
$$

with $R a$ the Rayleigh number on scale $L$ :

$$
R a=\frac{g A L^{3}}{\nu \kappa} .
$$

2. Numerical evidence [22] shows that the $\bar{V}(t)$ is indeed an increasing function of $\lambda$ and $\rho$.

\section{Nonlinear stability of planar fronts in narrow do- mains}

In this section we consider the reaction-diffusion Boussinesq problem (2.16) - (2.18) in a narrow domain, i.e. for small aspect ratio $\lambda$. The nonlinearity $f$ is of either one of the three types: KPP, ignition or bistable. We keep the time and space units chosen above (2.11, 2.13, 2.14, 2.15). We prove two results. The first one concerns traveling solutions of the form

$$
T(x, z, t)=T(x, z-c t), \quad v(x, z, t)=v(x, z-c t) .
$$

The result states that, if the Rayleigh number $\rho$ is sufficiently small, then such solutions must be planar fronts. Planar fronts are solutions of the form

$$
T(x, z, t)=P(z-c t), \quad v(x, z, t)=0
$$

for which $T$ does not depend on $x$ and $v$ vanishes. Planar fronts do exist for $c \geq 2$ in the KPP nonlinearity case and for a unique front speed $c_{*}$ in the ignition and bistable cases. 
Theorem 2 There exist constants $C_{1}>0$ and $C_{2}>0$ such that if $\lambda<C_{1}$, and $\rho<C_{2} / \lambda^{3}$, then the only solutions of (2.18)-(2.18), (2.21, 2.29) of traveling front type (4.1), are planar fronts of the form (4.9).

The second result in this section is about arbitrary solutions. We show that all solutions of the Boussinesq system in a narrow domain become eventually planar:

Theorem 3 There exist constants $C_{1}>0$ and $C_{2}>0$ so that if $\lambda<C_{1}$ and $\rho<C_{2} / \lambda^{3}$, then

$$
\|\omega(\cdot, t)\|_{L^{2}}+\left\|T_{x}(\cdot, t)\right\|_{L^{2}} \rightarrow 0 \text { as } t \rightarrow+\infty .
$$

Moreover, the front speed is uniformly bounded:

$$
\limsup _{t \rightarrow+\infty} \bar{V}(t) \leq 2
$$

Proof of Theorem 2. Let $T(x, z-c t), v(x, z-c t)$ be a traveling front solution of (2.16)(2.18), then

$$
\begin{aligned}
& -c T_{z}+v \cdot \nabla T=\Delta T+f(T) \\
& -c v_{z}+v \cdot \nabla v+\nabla p=\sigma \Delta v+\sigma \rho T \hat{e}_{z} \\
& -c \omega_{z}+v \cdot \nabla \omega=\sigma \Delta \omega+\sigma \rho T_{x} .
\end{aligned}
$$

We multiply the second equation in (4.5) by $v$ and integrate to obtain

$$
\sigma\|\nabla v\|^{2}=\sigma \rho \int T(x, z) w(x, z) d x d z
$$

In this section we will use the plain $L^{2}$ norm

$$
\|g\|^{2}=\int_{D}|g(x, z)|^{2} d x d z
$$

The right side may be bounded as in the proof of Lemma to obtain

$$
\sigma\|\nabla v\|^{2}=\sigma \rho \int T(x, z) w(x, z) d x d z \leq \sigma \rho\|T-\bar{T}\|\|w\| \leq \sigma \rho \lambda\left\|T_{x}\right\|\|w\|,
$$

where

$$
\bar{T}(z)=\int_{0}^{\lambda} T(x, z, t) \frac{d x}{\lambda} .
$$

We used above the fact that $w(x, z)$ has mean zero in $x$ because of incompressibility and boundary conditions at $z \rightarrow \infty$. Furthermore, it satisfies the Poincaré inequality so that $\|w\| \leq C \lambda\|\nabla v\|$, and the incompressibility of $v$ implies that $\|\nabla v\|=\|\omega\|$. Therefore (4.6) implies that

$$
\|w\| \leq C \rho \lambda^{3}\left\|T_{x}\right\|
$$

and

$$
\|\omega\| \leq C \rho \lambda^{2}\left\|T_{x}\right\| .
$$


We differentiate the first equation in (4.5) in $x$, multiply by $T_{x}$ and integrate to obtain:

$$
-\int T_{x}\left(v_{x} \cdot \nabla T\right) d x d z=\int\left|\nabla T_{x}\right|^{2} d x d z-\int f^{\prime}(T) T_{x}^{2} d x d z .
$$

We use the Poincaré inequality for $T_{x}$ to obtain a bound for the right side:

$$
\int\left|\nabla T_{x}\right|^{2} d x d z-\int f^{\prime}(T) T_{x}^{2} d x d z \geq\left(1-C \lambda^{2}\right) \int\left|\nabla T_{x}\right|^{2} d x d z \geq \frac{1}{2}\left\|\nabla T_{x}\right\|^{2}
$$

if $\lambda \leq C_{1}$ is sufficiently small, with $C_{1}$ an absolute constant that may depend only on $m_{1}=$ $\max _{0 \leq T \leq 1}\left|f^{\prime}(T)\right|$. The left side of (4.8) is bounded by

$$
\left|\int T_{x}\left(v_{x} \cdot \nabla T\right) d x d z\right|=\left|\int T\left(v_{x} \cdot \nabla T_{x}\right) d x d z\right| \leq\left\|v_{x}\right\|\left\|\nabla T_{x}\right\| \leq\|\omega\|\left\|\nabla T_{x}\right\| .
$$

The last two bounds imply that $\left\|\nabla T_{x}\right\| \leq 2\|\omega\|$ for a sufficiently small $\lambda$. Then the inequality (4.7) and the Poincaré inequality for $T_{x}$ imply that

$$
\left\|\nabla T_{x}\right\| \leq C \rho \lambda^{2}\left\|T_{x}\right\| \leq C \rho \lambda^{3}\left\|\nabla T_{x}\right\| .
$$

Therefore there exists a constant $C_{0}>0$ such that no $x$-dependent traveling front exists if

$$
\rho \leq \frac{C_{0}}{\lambda^{3}} .
$$

This finishes the proof of Theorem 2. We now prove Theorem 3 .

Proof of Theorem 3. We multiply the evolution equation for $v(x, z, t)$ by $v=(u, w)$ and integrate to obtain

$$
\frac{1}{2} \frac{d}{d t}\|v(\cdot, t)\|^{2}+\sigma\|\nabla v(\cdot, t)\|^{2}=\sigma \rho \int T(x, z, t) w(x, z, t) d x d z .
$$

The right side of the above equation may be bounded as in (4.6). We integrate (4.10) in time, and use (4.6) and the Poincaré inequality for $w(t, x, z)$ to get

$$
\begin{aligned}
& \|w(\cdot, t)\|^{2}+\frac{C \sigma}{\lambda^{2}} \int_{0}^{t}\|w(\cdot, s)\|^{2} d s \leq \sigma \rho \lambda \int_{0}^{t}\left\|T_{x}(\cdot, s)\right\|\|w(\cdot, s)\| d s+\|v(\cdot, 0)\|^{2} \\
& \leq \sigma \rho \lambda\left(\int_{0}^{t}\left\|T_{x}(\cdot, s)\right\|^{2} d s\right)^{1 / 2}\left(\int_{0}^{t}\|w(\cdot, s)\|^{2} d s\right)^{1 / 2}+\|v(\cdot, 0)\|^{2} \\
& \leq \frac{\sigma \rho \lambda}{2}\left(\frac{C}{\rho \lambda^{3}} \int_{0}^{t}\|w(\cdot, s)\|^{2} d s+\frac{\rho \lambda^{3}}{C} \int_{0}^{t}\left\|T_{x}(\cdot, s)\right\|^{2} d s\right)+\|v(\cdot, 0)\|^{2}
\end{aligned}
$$

Therefore we have

$$
\int_{0}^{t}\|w(\cdot, s)\|^{2} d s \leq C \rho^{2} \lambda^{6} \int_{0}^{t}\left\|T_{x}(\cdot, s)\right\|^{2} d s+\frac{C \lambda^{2}}{\sigma}\|v(\cdot, 0)\|^{2} .
$$

Furthermore, once again, after integrating (4.10) in time we obtain

$$
\int_{0}^{t}\|\omega(\cdot, s)\|^{2} d s \leq \rho \lambda \int_{0}^{t}\left\|T_{x}(\cdot, s)\right\|\|w(s)\| d s \leq C \rho^{2} \lambda^{4} \int_{0}^{t}\left\|T_{x}(s)\right\|^{2} d s+\frac{C}{\sigma}\|v(\cdot, 0)\|^{2} .
$$


Furthermore, we differentiate the first equation in (2.17) in $x$ and obtain

$$
T_{x t}+v \cdot \nabla T_{x}+v_{x} \cdot \nabla T=\Delta T_{x}+f^{\prime}(T) T_{x} .
$$

Then, as in the proof of Theorem 2, provided that $\lambda<C_{1}$ is sufficiently small, if we multiply (4.13) by $T_{x}$, and integrate, we obtain, using the Poincaré inequality for $T_{x}$ :

$$
\frac{1}{2} \frac{d}{d t}\left(\left\|T_{x}\right\|^{2}\right)+C\left\|\nabla T_{x}\right\|^{2} \leq\left\|v_{x}\right\|\left\|\nabla T_{x}\right\|
$$

This implies that

$$
\frac{1}{2} \frac{d}{d t}\left(\left\|T_{x}\right\|^{2}\right)+\frac{C^{\prime}}{2 \lambda^{2}}\left\|T_{x}\right\|^{2} \leq \frac{C^{\prime \prime}}{2}\|\omega\|^{2}
$$

and hence

$$
\left\|T_{x}(\cdot, t)\right\|^{2} \leq\left\|T_{x}(\cdot, 0)\right\|^{2} e^{-C^{\prime} t / \lambda^{2}}+C^{\prime \prime} \int_{0}^{t}\|\omega(\cdot, s)\|^{2} e^{-C^{\prime}(t-s) / \lambda^{2}} d s .
$$

We integrate (4.14) and obtain

$$
\begin{aligned}
& \int_{0}^{t}\left\|T_{x}(\cdot, s)\right\|^{2} d s \leq C \lambda^{2}\left\|T_{x}(\cdot, 0)\right\|^{2}+C \int_{0}^{t} \int_{0}^{s}\left\|\omega\left(\cdot, s_{1}\right)\right\|^{2} e^{-C^{\prime}\left(s-s_{1}\right) / \lambda^{2}} d s_{1} d s \\
& \leq C \lambda^{2}\left\|T_{x}(\cdot, 0)\right\|^{2}+C \lambda^{2} \int_{0}^{t}\|\omega(\cdot, s)\|^{2} d s .
\end{aligned}
$$

We use now the bound (4.12) to get

$$
\int_{0}^{t}\left\|T_{x}(\cdot, s)\right\|^{2} d s \leq C \lambda^{2}\left\|T_{x}(\cdot, 0)\right\|^{2}+\frac{C \lambda^{2}}{\sigma}\|v(\cdot, 0)\|^{2}+C \rho^{2} \lambda^{6} \int_{0}^{t}\left\|T_{x}(s)\right\|_{2}^{2} d s .
$$

Therefore there exists a constant $C_{0}>0$ so that if (4.9) holds, then

$$
\int_{0}^{t}\left\|T_{x}(\cdot, s)\right\|^{2} d s \leq C \lambda^{2}\left\|T_{x}(\cdot, 0)\right\|^{2}+\frac{C \lambda^{2}}{\sigma}\|v(\cdot, 0)\|^{2} .
$$

Then (4.9) and (4.12) imply that

$$
\begin{gathered}
\int_{0}^{t}\|\omega(\cdot, s)\|^{2} d s \leq C \rho^{2} \lambda^{4}\left[C \lambda^{2}\left\|T_{x}(\cdot, 0)\right\|^{2}+\frac{C \lambda^{2}}{\sigma}\|v(\cdot, 0)\|^{2}\right]+\frac{C}{\sigma}\|v(\cdot, 0)\|^{2} \\
\leq C\left\|T_{x}(\cdot, 0)\right\|^{2}+\frac{C}{\sigma}\|v(\cdot, 0)\|^{2} .
\end{gathered}
$$

The bounds (4.14) and (4.16) together imply in an elementary way that

$$
\left\|T_{x}(\cdot, t)\right\| \rightarrow 0 \text { as } t \rightarrow+\infty \text {. }
$$

We multiply the vorticity equation (2.16) by $\omega$ and integrate in space:

$$
\frac{1}{2} \frac{d}{d t}\left(\|\omega(\cdot, t)\|^{2}\right)+\|\nabla \omega(\cdot, t)\|^{2} \leq \sigma \rho\|\omega(\cdot, t)\|\left\|T_{x}(\cdot, t)\right\| .
$$


Then we have

$$
\begin{aligned}
\|\omega(\cdot, t)\|^{2} & \leq\|\omega(\cdot, \tau)\|^{2}+2 \sigma \rho \int_{\tau}^{t}\|\omega(\cdot, s)\|\left\|T_{x}(\cdot, s)\right\| d s \\
& \leq\|\omega(\cdot, \tau)\|^{2}+2 \sigma \rho\left(\int_{\tau}^{\infty}\|\omega(\cdot, s)\|^{2} d s\right)^{1 / 2}\left(\int_{\tau}^{\infty}\left\|T_{x}(\cdot, s)\right\|^{2} d s\right)^{1 / 2} .
\end{aligned}
$$

The uniform bound (4.16) implies that there exists a sequence of times $\tau_{k} \rightarrow+\infty$ so that $\left\|\omega\left(\cdot, \tau_{k}\right)\right\| \rightarrow 0$. Then we have for $t>\tau_{k}$ :

$$
\|\omega(\cdot, t)\|^{2} \leq\left\|\omega\left(\cdot, \tau_{k}\right)\right\|^{2}+2 \sigma \rho\left(\int_{\tau_{k}}^{\infty}\|\omega(\cdot, s)\|^{2} d s\right)^{1 / 2}\left(\int_{\tau_{k}}^{\infty}\left\|T_{x}(\cdot, s)\right\|^{2} d s\right)^{1 / 2}
$$

and hence $\|\omega(\cdot, t)\| \rightarrow 0$ as $t \rightarrow+\infty$ because of (4.15) and (4.16). In order to prove (4.4) we note that (4.17) implies that

$$
\int_{t_{1}}^{t_{2}}\|\nabla \omega(\cdot, s)\|^{2} d s \leq \int_{t_{1}}^{t_{2}} \sigma \rho\|\omega(\cdot, s)\|\left\|T_{x}(\cdot, s)\right\| d s+\frac{1}{2}\left\|\omega\left(\cdot, t_{1}\right)\right\|^{2}
$$

so that (4.3) implies that for any $\varepsilon>0$ we have for $t_{1}, t_{2}>t_{1}+1$ sufficiently large we have

$$
\int_{t_{1}}^{t_{2}}\|\nabla \omega(\cdot, s)\|^{2} d s \leq \varepsilon\left(t_{2}-t_{1}\right)
$$

Therefore, (3.11), (3.21) and the Cauchy-Schwartz inequality imply that

$$
\bar{V}(t) \leq 2+\frac{1}{t} \int_{0}^{t}\|w(s)\|_{\infty} d s+o(1) \leq 2+\frac{C}{\sqrt{t}}\left(\int_{0}^{t}\|\nabla \omega(\cdot, s)\|^{2} d s\right)^{1 / 2}+o(1) \leq 2+\varepsilon+o(1)
$$

and (4.4) follows. This finishes the proof of Theorem 3 .

\section{$5 \quad$ Linear instability}

In the previous section we established the stability of planar fronts with respect to short wavelength perturbations. We analyze now the linear instability of planar front with respect to long wavelength perturbations.

We perform a Galilean transformation $z \mapsto z-v_{0} t$ in (2.1)-(2.3) following the flat front. We write $T(x, z, t)=\tau\left(z-v_{0} t\right)+\theta\left(x, z-v_{0} t, t\right)$, and, with a slight abuse of notation, $v(x, z, t)=v\left(x, z-v_{0} t, t\right)$. We also linearize equations (2.1)-(2.3), dropping the terms that are quadratic in $\theta$ and $v$. We obtain the linearized system

$$
\begin{aligned}
& \frac{\partial \theta}{\partial t}-v_{0} \partial_{z} \theta-\kappa \Delta \theta-\frac{v_{0}^{2}}{4 \kappa} f^{\prime}(\tau(z)) \theta=-w \tau^{\prime} \\
& \frac{\partial u}{\partial t}-v_{0} \partial_{z} u-\nu \Delta u+\nabla p=g A \theta e_{z}
\end{aligned}
$$

and for the vorticity we get the equation

$$
\frac{\partial \omega}{\partial t}-v_{0} \partial_{z} \omega-\nu \Delta \omega=g A \partial_{x} \theta
$$


Using the same reaction units (2.11)-(2.15) as before, equations (5.1), (5.3) become

$$
\frac{\partial \theta}{\partial t}-2 \frac{\partial \theta}{\partial z}-\Delta \theta-f^{\prime}(P(z)) \theta=-w P^{\prime}(z)
$$

and

$$
\frac{\partial \omega}{\partial t}-2 \frac{\partial \omega}{\partial z}-\sigma \Delta \omega=\sigma \rho \frac{\partial \theta}{\partial x}
$$

The nonlinear equations, in the same units and frame of reference, are

$$
\frac{\partial \omega}{\partial t}+v \cdot \nabla \omega-2 \frac{\partial \omega}{\partial z}-\sigma \Delta \omega=\sigma \rho \frac{\partial T}{\partial x}
$$

and

$$
\frac{\partial T}{\partial t}+v \cdot \nabla T-2 \frac{\partial T}{\partial z}-\Delta T=f(T)
$$

We wish to show that flat profiles cannot remain linearly stable with respect to sufficiently long wave infinitesimal perturbations. We start for simplicity with the case of the infinite Prandtl number. We retain (5.4) but we use the infinite Prandtl number limit of (5.5),

$$
-\Delta \omega=\rho \frac{\partial \theta}{\partial x}
$$

which, together with (2.18) allows us to write the active scalar rule

$$
w=-\rho\left(\partial_{x}\right)^{2}(-\Delta)^{-2} \theta .
$$

We express $\theta(x, z, t)$ in terms of its Fourier series:

$$
\theta(x, z, t)=\sum_{k \in \frac{2 \pi}{\lambda} \mathbf{Z}} g_{k}(z, t) e^{i k x} .
$$

The linearized temperature equation (5.4) transforms into

$$
\frac{\partial g_{k}}{\partial t}-2 \frac{\partial g_{k}}{\partial z}+\left(k^{2}-\partial_{z z}\right) g_{k}-f^{\prime}(P) g_{k}=\rho Q K g_{k}
$$

with $k= \pm \frac{2 \pi}{\lambda}, \pm 2 \frac{2 \pi}{\lambda}, \ldots$, the operator $K$ defined by the Fourier transform of (5.9)

$$
K g=k^{2}\left(k^{2}-\partial_{z z}\right)^{-2} g
$$

and

$$
Q(z)=-P^{\prime}(z)>0 .
$$

We will choose the smallest positive wave number from now on:

$$
k=\frac{2 \pi}{\lambda} .
$$

The operator $K$ defined by (5.12) is given explicitly by a convolution with a positive function

$$
(K g)(z)=\frac{1}{4 k} \int_{-\infty}^{\infty}(1+k|z-\zeta|) e^{-k|z-\zeta|} g(\zeta) d \zeta .
$$


The expression (5.15) is obtained by an elementary calculation, starting from

$$
\left(k^{2}-\partial_{z z}\right)^{-1} g(z)=\frac{1}{2 k} \int_{-\infty}^{\infty} e^{-k|z-\zeta|} g(\zeta) d \zeta
$$

and iterating. It is well known that the profile $P$ is decreasing in the case of KPP, bistable and ignition nonlinearities [24], so that the function $Q$ is positive. Moreover

$$
Q(z) \geq a e^{-b|z|}
$$

holds for all $z$, with $a>0$ and $b>0$ absolute numbers that depend only on the nonlinearity $f(T)$. The positivity of $Q$ and of the kernel of the operator $K$ in the right hand side of (5.11) imply that the solution of the initial value problem (5.11) remains nonnegative if the initial data is nonnegative. Let us consider a function $\phi(z)$ which has the properties

$$
e^{-k|z|} \leq \phi(z) \leq C e^{-k|z|}
$$

with $C>1$ and

$$
\left|\phi^{\prime}(z)\right| \leq C k e^{-k|z|}, \quad\left|\phi^{\prime \prime}(z)\right| \leq C k^{2} e^{-k|z|} .
$$

Such a function is obtained for instance by gluing smoothly $3 e^{1-z}$ on $z \geq 1$ to $3 e^{1+z}$ on $z \leq-1$ by a function bounded below by 2 on the interval $[-1,1]$. Then one rescales $z \mapsto k z$. We multiply the equation (5.11) by $\phi(z)$ and integrate. Using the properties of $\phi$ and the positivity of $g_{k}(z, t)$ we obtain

$$
\frac{d}{d t} \int \phi(z) g_{k}(z, t) d z \geq-M \int \phi(z) g_{k}(z) d z+\rho \int \phi(z) Q(z)\left(K g_{k}\right)(z) d z
$$

This follows from integration by parts, and bounds (5.19) on the first two derivatives of $\phi$ by constant multiples of $\phi$. The constant $M \leq 2 C\left(1+k+k^{2}\right)$, so it is uniformly bounded for bounded $k$, for instance for $0 \leq k \leq 1$. Now we bound from below the term

$$
\rho \int \phi(z) Q(z)\left(K g_{k}\right)(z) d z \geq \frac{\rho}{4 k} \iint a e^{-b|z|} \phi(z) e^{-k|z|-k|\zeta|} g_{k}(\zeta, t) d \zeta d z
$$

We used (5.17), (5.15) and the positivity of $g_{k}$. We neglected the nonnegative term contributed by $k|z-\zeta|$ in (5.15). Now we use (5.18):

$$
\rho \int \phi(z) Q(z)\left(K g_{k}\right)(z) d z \geq \frac{\rho}{4 k} \frac{a}{C}\left(\int e^{-(b+2 k)|z|} d z\right)\left(\int \phi(\zeta) g_{k}(\zeta, t) d \zeta\right) \text {. }
$$

We obtain the ordinary differential inequality

$$
\frac{d}{d t} \int \phi(z) g_{k}(z) d z \geq\left(\frac{\rho}{4 k} \frac{a}{2 C(b+2 k)}-M\right) \int \phi(z) g_{k}(z) d z
$$

and thus $\left\|g_{k}\right\|_{L^{1}(\mathbb{R})}$ grows exponentially in time. Therefore we have the following theorem for the infinite Prandtl number case: 
Theorem 4 Let $P(z-2 t), u=0$ be a planar, $x$-independent traveling front solution of the infinite Prandtl number Boussinesq system

$$
\frac{\partial T}{\partial t}+v \cdot \nabla T-\Delta T=f(T)
$$

with

$$
-\Delta v+\nabla p=\rho T e_{z}, \quad \nabla \cdot u=0,
$$

with front boundary conditions for $T$ at $z= \pm \infty$, vanishing velocity at $z= \pm \infty$ and periodic boundary conditions in $x$ of period $\lambda$. There exists a positive constant $\beta>0$ such that, if

$$
\rho \lambda>\beta
$$

then the solution $P$ is linearly unstable. This means that there exist infinitesimal perturbations which grow exponentially, when viewed in a Galilean frame of reference moving with the traveling front. Their exponential growth rate is proportional to $\rho \lambda$.

A similar instability analysis may be applied to a convective system with the infinite Prandtl number equation (5.8) replaced by the Darcy law that was studied numerically in [10].

We return to the system (5.4, 5.5) at a finite Prandtl number. We use the Fourier expansion

$$
w(x, z, t)=\sum_{n \in \mathbf{Z}} w_{k}(z, t) e^{2 \pi i n x / \lambda}
$$

and using (2.18) we have, with $k=2 \pi n / \lambda$,

$$
w_{k}(z, t)=-\frac{i}{2} \int e^{-k\left|z-z^{\prime}\right|} \omega_{k}\left(z^{\prime}, t\right) d z^{\prime}
$$

with the obvious notation for the Fourier coefficients of $\omega$. An elementary calculation involving solving the linear equation (5.5) starting with the zero initial data $\omega(x, z, 0)=0$, and using (2.18), gives

$$
w_{k}(z, t)=\frac{\sigma \rho k}{2} \int_{0}^{t} \int g_{k}\left(z_{2}, s\right) I_{k}\left(z-z_{2}+2(t-s), t-s\right) d z_{2} d s
$$

where $g_{k}(z, t)$ are the Fourier coefficients of $\theta$ as in (5.10) and

$$
I_{k}(\zeta, \tau)=e^{-\sigma k^{2} \tau} \int e^{-k|\zeta+u \sqrt{2 \sigma \tau}|} e^{-\frac{|u|^{2}}{2}} \frac{d u}{\sqrt{2 \pi}} .
$$

We now bound $I_{k}$ at this point as follows:

$$
I_{k}(\zeta, \tau) \geq e^{-\sigma k^{2} \tau} \int e^{-k|\zeta|-k|u| \sqrt{2 \sigma \tau}} e^{-\frac{|u|^{2}}{2}} \frac{d u}{\sqrt{2 \pi}} \geq 2 e^{-k|\zeta|} \operatorname{Erf}(k \sqrt{2 \sigma \tau})
$$

with

$$
\operatorname{Erf}(a)=\int_{a}^{\infty} e^{-\frac{u^{2}}{2}} \frac{d u}{\sqrt{2 \pi}}
$$


For $a \geq 1$ we will use just a piece of this integral,

$$
\operatorname{Erf}(a) \geq \int_{a}^{2 a} e^{-\frac{u^{2}}{2}} \frac{d u}{\sqrt{2 \pi}} \geq \frac{1}{\sqrt{2 \pi}} e^{-a^{2}}
$$

and for $a \leq 1$ we write

$$
\operatorname{Erf}(a) \geq \operatorname{Erf}(1)
$$

This allows us to deduce, for later use

$$
I_{k}(\zeta, \tau) \geq 2\left(1-\frac{1}{\sqrt{2 \pi}}\right) e^{-k|\zeta|} e^{-2 \sigma k^{2} \tau}
$$

The evolution equation for $g_{k}$ follows from (5.4),

$$
\frac{\partial g_{k}}{\partial t}-2 \frac{\partial g_{k}}{\partial z}+\left(k^{2}-\partial_{z z}\right) g_{k}-f^{\prime}(P) g_{k}=\sigma \rho Q w_{k}
$$

with $w_{k}$ computed using (5.29) and with $Q=-P^{\prime}$ as before. Because of the explicit expression (5.29) we see that if the initial data $g_{k}(z, 0)$ is real and positive, then $g_{k}(z, t)$ remains real and positive. We take such initial data. Then, using (5.35) and the triangle inequality we get,

$$
w_{k}(z, t) \geq\left(1-\frac{1}{\sqrt{2 \pi}}\right) \rho \sigma k e^{-k|z|} \int_{0}^{t} e^{-2(t-s) k(\sigma k+2)}\left[\int g_{k}\left(z_{2}, s\right) e^{-k\left|z_{2}\right|} d z_{2}\right] d s .
$$

As in the case of the infinite Prandtl number, we take a function $\phi(z)$ with properties (5.18), (5.19), multiply (5.36) by $\phi$ and integrate. Let us denote

$$
Y(t)=\int \phi(z) g_{k}(z, t) d z
$$

Integrating by parts we get

$$
\frac{d Y}{d t}+C_{3}\left(1+k+k^{2}\right) Y \geq C_{4} \sigma \rho k \int_{0}^{t} e^{-2(t-s) k(\sigma k+2)} Y(s) d s .
$$

with $C_{3}$ and $C_{4}$ positive numbers that depend on the properties of $\phi$. Consider also

$$
Z(t)=e^{2 k(\sigma k+2) t} Y(t) \text {. }
$$

Multiplying by $e^{2 k(\sigma k+2) t}$, we get from (5.39)

$$
\frac{d Z}{d t}+\beta Z \geq \alpha \int_{0}^{t} Z(s) d s
$$

with

$$
\beta=C_{3}\left(1+k+k^{2}\right)-2 k(\sigma k+2)
$$

and

$$
\alpha=C_{4} \sigma \rho k
$$


It is clear that solutions of the differential inequality (5.41) are larger than solutions of the ODE

$$
\frac{d y}{d t}+\beta y=\alpha \int_{0}^{t} y(s) d s
$$

with the same initial data. This ODE is solved differentiating one more time,

$$
\frac{d^{2} y}{d t^{2}}+\beta \frac{d y}{d t}-\alpha y=0
$$

and seeking solutions of the form $e^{\mu t}$. Equation (5.45) always has at least one exponentially growing solution because $\alpha>0$ (irrespective of the sign of $\beta$ ). The general solution of (5.45) is

$$
y(t)=y_{1} e^{t \mu_{+}}+y_{2} e^{t \mu_{-}}
$$

with

$$
\mu_{ \pm}=\frac{1}{2}\left(-\beta \pm \sqrt{\beta^{2}+4 \alpha}\right)
$$

The solutions of (5.44) correspond to the linear subspace for which $\frac{d y}{d t}(0)+\beta y(0)=0$. Choosing without loss of generality the coefficient of the growing exponential $y_{1}=1$, we deduce the relation

$$
\mu_{+}+\beta+y_{2}\left(\mu_{-}+\beta\right)=0
$$

In order to have exponentially growing positive solutions we need the initial datum for (5.44) to be positive. This initial datum is $1+y_{2}$ and is computed using (5.47) and (5.48)

$$
1+y_{2}=\frac{2 \sqrt{\beta^{2}+4 \alpha}}{\sqrt{\beta^{2}+4 \alpha}-\beta}
$$

so it is always positive. Starting with this initial condition (or any positive multiple thereof) we get exponential growth for $Z(t)$. This will imply exponential growth for $Y(t)$ if

$$
\mu_{+}>2 k(\sigma k+2)
$$

This turns out to be the condition

$$
C_{4} \sigma \rho>2 C_{3}\left(1+k+k^{2}\right)(\sigma k+2)
$$

or, putting $C=\frac{2 C_{3}}{C_{4}}$,

$$
\rho>C\left(\frac{2}{\sigma}+k\right)\left(1+k+k^{2}\right)
$$

Theorem 5 Planar reactive Boussinesq fronts (5.6, 5.7) are linearly unstable to large wavelength perturbations whenever the local Rayleigh number $\rho$ based on the laminar front thickness is large compared to the inverse of the Prandtl number,

$$
\rho>\frac{2 C}{\sigma} .
$$

Perturbations with wave numbers $k$ satisfying (5.59) grow exponentially in a frame of reference moving with the planar front. The growth rate is proportional to $\sqrt{\sigma \rho k}$. 
Remark. The exponential growth rate proportional to the square root of the wave number is a signature of the Rayleigh-Taylor instability, operating here only at large scales. When the wave length of the initial perturbation is decreased to a length comparable to the thickness of the planar front, the perturbation decays in time.

\section{Conclusions}

We proved that the reactive Boussinesq system in a strip has front solutions with bounded speeds, if the nonlinearity is of concave KPP type. The bounds grow with the aspect ratio, but nevertheless, front acceleration does not occur in this system. The concavity of the nonlinearity was used in the proof. We do not know at present whether the divergence of the bound with aspect ratio is sharp. For small aspect ratios and for small Rayleigh numbers, the only traveling modes are planar, and all front-like solutions become planar. This result is expected to be valid in more generality than proved here. For large enough Rayleigh numbers, if the aspect ratio is large, then the planar fronts lose stability to long wave perturbations. The instability is of Rayleigh-Taylor type. This again is expected to hold in greater generality than proved here. The results proved here agree qualitatively with the recent numerical study [22].

Acknowledgments The authors thank N. Vladimirova and R. Rosner for extensive scientific interactions. PC was supported partially by NSF DMS-0202531. AK was supported partially by NSF grant DMS-0102554 and by an Alfred P. Sloan Research Fellowship. LR was supported partially by NSF grant DMS-0203537 and by an Alfred P. Sloan Research Fellowship. This research is supported in part by the ASCI Flash center at the University of Chicago under DOE contract B341495.

\section{References}

[1] H. Berestycki, The influence of advection on the propagation of fronts in reaction-diffusion equations, Proceedings NATO ASI Conf. Cargese, H. Berestycki and Y. Pomeau eds. Kluwer (to appear).

[2] H. Berestycki and F. Hamel, Front propagation in periodic excitable media, Comm. Pure Appl. Math. 55, 2002, 949-1032.

[3] H. Berestycki, B. Larrouturou and P. L. Lions, Multi-dimensional traveling wave solutions of a flame propagation model, Arch. Rational Mech. Anal., 111, 1990, 33-49.

[4] H. Berestycki and L. Nirenberg, Traveling fronts in cylinders, Annales de l'IHP, Analyse non linéare, 9, 1992, 497-572.

[5] P. Constantin, A. Kiselev, A. Oberman, L. Ryzhik, Bulk burning rate in passive-reactive diffusion, Arch. Rat. Mech. Anal. 154, 2000, 53-91.

[6] P. Constantin, A. Kiselev, L. Ryzhik, Quenching of flames by fluid advection, Comm. Pure Appl. Math 54, 2001, 1320-1342. 
[7] S. Chandrasekhar, Hydrodynamic and hydromagnetic stability, Clarendon Press 1961.

[8] J. Daou and M. Matalon, Flame propagation in Poiseuille flow under adiabatic conditions, Combustion and Flame, 124, 2001, 337-349.

[9] J. Daou and M. Matalon, Influence of conductive heat-losses on the propagation of premixed flames in channels, Combustion and Flame 128, 2001, 321-339.

[10] A. de Wit, Fingering of chemical fronts in porous media, Phys. Rev. Let., 87, 054502.

[11] M. Freidlin and J. Gärtner, On the propagation of concentration waves in periodic and random media, Soviet Math. Dokl., 20, 1979, 1282-1286.

[12] M. Freidlin, Geometric optics approach to reaction-diffusion equations, SIAM J. Appl. Math., 46, 1986, 222-232.

[13] S. Heinze, G. Papanicolau and A. Stevens, Variational principles for propagation speeds in inhomogeneous media, SIAM J. Appl. Math. 62, 2001, 129-148.

[14] L. Kagan and G. Sivashinsky, Flame propagation and extinction in large-scale vortical flows, Combust. Flame 120, 2000, 222-232.

[15] L. Kagan, P.D. Ronney and G. Sivashinsky, Activation energy effect on flame propagation in large-scale vortical flows, Combust. Theory Modelling 6, 2002, 479-485.

[16] A. Kiselev and L. Ryzhik, Enhancement of the travelling front speeds in reaction-diffusion equations with advection, Ann. Inst. H. Poincaré Anal. Non Linéaire 18, 2001, 309-358.

[17] A. Majda and P. Souganidis, Large scale front dynamics for turbulent reaction-diffusion equations with separated velocity scales, Nonlinearity, 7, 1994, 1-30.

[18] S. Malham and J. Xin, Global solutions to a reactive Boussinesq system with front data on an infinite domain, Comm. Math. Phys., 193, 1998, 287-316.

[19] N. Peters, Turbulent Combustion, Cambridge University Press, Cambridge, UK 2000.

[20] R. Texier-Picard and V. Volpert, Problèmes de réaction-diffusion-convection dans des cylindres non bornés, C. R. Acad. Sci. Paris Sr. I Math. 333, 2001, 1077-1082

[21] R. Texier-Picard and V. Volpert, Reaction-diffusion-convection problems in unbounded cylinders, Preprint 2001.

[22] N. Vladimirova, R. Rosner, Model flames in the Boussinesq limit: the effects of feedback, Preprint, 2002.

[23] N. Vladimirova, P. Constantin, A. Kiselev, O. Ruchayskiy and L. Ryzhik, in preparation.

[24] A. Volpert, V. Volpert and V. Volpert, Travelling Wave Solutions of Parabolic Systems, Translations of mathematical Monographs, 140, Amer. Math. Soc., Providence, Rhode Island 1994. 
[25] J. Xin, Existence of planar flame fronts in convective-diffusive periodic media, Arch. Rat. Mech. Anal., 121, 1992, 205-233.

[26] J. Xin, Existence and nonexistence of travelling waves and reaction-diffusion front propagation in periodic media, Jour. Stat. Phys., 73, 1993, 893-926.

[27] J. Xin, Analysis and modelling of front propagation in heterogeneous media, SIAM Rev., 42, 2000, 161-230.

[28] Ya.B. Zeldovich, G.I. Barenblatt, V.B. Librovich and G.M. Makhviladze, The Mathematical Theory of Combustion and Explosions, Translated from the Russian by Donald H. McNeill. Consultants Bureau [Plenum], New York, 1985. 